\title{
Factors Affecting the Bond Strength between Denture Basis and the Soft Relining Materials - A Review
}

\author{
Ilian Hristov ${ }^{1}$, Diyan Slavchev ${ }^{2}$, Boyan Pavlov $^{3}$, Stoyan Yankov ${ }^{4}$, Rangel Todorov ${ }^{5}$ \\ ${ }^{1,2,3,4}$ Department of Prosthodontics, Faculty of Dental Medicine, Medical University, 15A Vasil Aprilov, Plovdiv 4000, Bulgaria \\ ${ }^{5}$ Department of Prosthodontics, Faculty of Dental Medicine, Medical University, 15 Akademik Ivan Evstratiev Geshov, Sofia 1431, \\ Bulgaria
}

\begin{abstract}
Problems of the patients with removable dentures usually are: poor adhesion and stability, pain, wounds, difficult adaptation to the new dentures etc. Use of soft relining materials (SRM) can overcome these difficulties to some extent. The problems with the soft relining materials are usually connected with: odor, hardening of the material, staining, but the problem with the bond between the two materials is of primary importance. This is the major obstacle, that makes a lot of clinicians to use them in their practices reluctantly. There are many factors that contribute to the bond strength between the materials. Their acquaintance is very important to overcome the disadvantages of SRM.
\end{abstract}

Key words: "two-layer" denture, soft relining materials

\section{Introduction}

Durability of a "two-layer" denture depends mainly on the bond strength between the two materials (denture basis and the soft relining material). Moodhy and Jagger emphasize the role of different methods for bond strength investigation [1]. Besides that, there are plenty of factors affecting the bond strength. Many research articles have been published so far, concerning this topic. The aim of this review is to reveal the different aspects of the problem with the bond strength between the denture basis and the SRM.

Types of tests, contributing to the bond strength determination

Various types of tests for evaluation of the bond strength are recommended $[2,3,4,5]$. Three of the most used ones are: tensile bond strength, peeling bond strength and shear bond strength tests [6], (fig. 1).

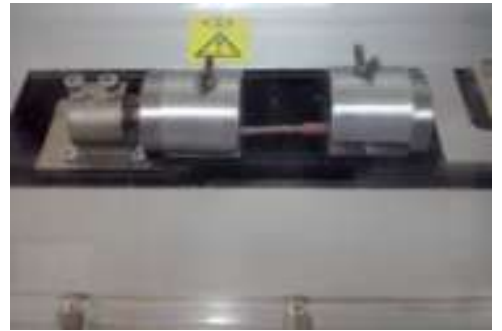

Figure 1: A device for tensile bond strength tests: LMT-100 /LAM Technologies/

Kendall and Lindley separately from each other apply the theory of the energy of breaking of elastic layer from a solid basis. According to this theory, the necessary energy for peeling depends not only on the bond surface energy, but on the elastic deformation of the object as well. The elasticity is exceptionally important for materials which withstand tension, reaching their module of elasticity without destroying even in small angles of peeling $[7,8]$.
Wright, $\mathrm{P}$. considers, that the nature of the occurred tension in denture periphery is characterized best with the peeling strength tests [9]. Rajaganesh and Al Rifaiy consider that shear bond strength tests present a clear idea of the clinical durability of the material $[10,11]$. Naik and Jabade consider, that tensile bond strength tests are effective enough, because they are similar to the action of the vertical forces of the masticatory act. Moreover, they present a cutting surface, giving information about the structure of the interface on the site of dismemberment. This statement is with agreement of Mutluay and Ruyter, who claim the same in their investigation $[12,13]$.

Influence of various immersion solutions on bond strength

The rupture energy depends on the nature of the material and the time of immersion in various kinds of fluids. Moreover, the velocity of the experiment and elastic properties of the materials are also important. Increase of the immersion time, decreases the values of rupture forces. Acryl based materials kept in distilled water present the highest values, while the silicone liners kept in disinfection solutions have the lowest values. In acryl materials, the tendency is for increasing of these levels between $4^{\text {th }}$ and $16^{\text {th }}$ week. Determination of rupture property is particularly important for SRM, because in mouth conditions, these materials have to withstand similar forces [14].

Water absorption considerably influences the bond strength and is a reason for dismemberment of the materials as well. Cohesion fractures are more frequent than adhesion ones in samples put under peeling tests. Increase in weight, especially in silicone materials up to $4 \%$ is established Using of scanning electronic microscopy (SEM) confirms reliability of the bond between acryl SRM and denture basis. Clean and smooth contact zone in silicone based materials is observed, which proves the hydrolysis of the adhesive with time [15]

Volume 6 Issue 8, August 2017

www.ijsr.net

Licensed Under Creative Commons Attribution CC BY 


\section{International Journal of Science and Research (IJSR) \\ ISSN (Online): 2319-7064}

Index Copernicus Value (2015): 78.96 | Impact Factor (2015): 6.391

Wright, P. investigates the shear bond strength of different silicone liners. He concludes that, after 6 months stay in distilled water, these materials possess the same strength, as the irreversible hydrocolloids. Braden first initiates this method in dentistry investigating rupture characteristics of impression materials. Later on, the method was developed by Webber and Ryge [16].

The medium where SRM are preserved has an enormous influence on their properties [17]. Acrylic materials exceed silicone ones at the beginning. It becomes evident that in the course of the experiments, silicone materials are slightly influenced by aging and heat-curing materials present better indices than the self-curing materials [18]. This data is in contradiction to those of Dootz and Emmer, who claim that immersion in water medium, bond strength of some materials increases. Vergani et al. also report that 30 days stay in water contributes to increase of the shear bond strength.

The investigations of different authors prove the influence of effervescent tablets for disinfection on bond strength, as well as their degree of infiltration. It turned out that Corega ${ }^{1}$ tablets don't render significant influence on bond strength, and infiltration degree is less in acrylic materials, compared to the silicone ones [19].

\section{Influence of chemical composition of denture basis and relining materials on bond strength}

The type of polymer of the denture basis, as well as the type of the relining material indisputably influence the bond strength. The results of the shear bond strength tests depend on the plasticizer used as well [20].

The best results are obtained when chemically homogenious materials are bonded to similar ones. The worst results are obtained when for example urethanedimethacrylate (UDMA) SRM and PMMA denture basis are combined. The highest frequencies of adhesive failures are in this combination of materials [21].

The role of the chemical composition of the denture basis and its reflection to the bond strength has been investigated by many authors. The bond strength between acrylic SRM and acrylic basis has the highest values, because of the similarity of their chemical composition. Cohesive failures normally predominate in these samples. Fowler, reports for significant differences in the bond strength between "Silastic 390 " and various PMMA denture basis, due to their different chemical composition [22]. Wood et al. consider that type of denture basis has no effect on bond strength [23].

Lassila et al. investigate the bond strength among several types SRM connected to glass fibers reinforced PMMA and classical PMMA (as a control group) [24]. Because the use of SRM leads to reduction of the thickness of denture basis, the opportunity for breaking is real (fig. 2). An adequate alternative is the use of glass fibers reinforced acrylic resins as well as resins with butadien stiren [25].

${ }^{1}$ Corega - GlaxoSmithKline

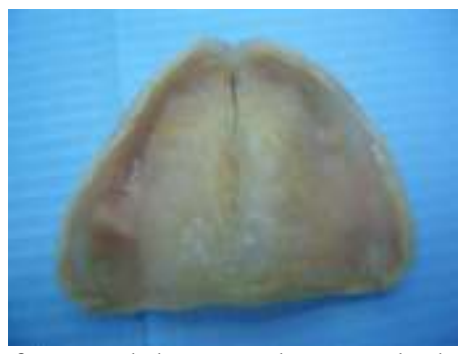

Figure 2: A fractured denture, due to a lack of thickness

Influence of various types of interface on bond strength A lot of experiments have been done, in order to interact the contact surface of the samples, aiming to increase the bond strength.

Tests were led in two directions:

- First - roughening of the contact surface using air abrasion (sand blasting)

- Second - reading the influence of various primers before and after thermo cycling process [26]

Storer, reports that the rough surface increases the bond strength almost twice. By roughening, the contact surface is enlarged and micromechanical retentions upon the interface are obtained. This statement is in contradiction to the results of Amin, Fletcher and Ritchi [27]. In their opinion, by roughening, the bond strength doesn't increase, but even has a reverse effect. A reason for that the authors find in the creation of micro cracks, lacunas and hollows, where before putting of SRM air bubbles are possible to be trapped and in this way the contact surface will be reduced.

Different experiments have been done, where the bond strength has been evaluated between heat-curing silicone material Molloplast B and three types of acrylic surfaces:

- prepolymerized PMMA (smooth surface),

- prepolymerized PMMA (putty consistency),

- PMMA, roughened by bur in advance

By roughening, higher values of bond strength are obtained. The explanation of the authors is in the created rough zones. Greater force is needed to make both contact surfaces to skid and tear. As far as the tensile bond strength values are concerned, they are the highest in the group of Molloplast B - PMMA (putty consistency).

Kawano et al. do a similar research. They use samples of SRM bonded to:

- polymerized PMMA

- unpolymerized PMMA.

They conclude, that the bond strength depends on the stage of polymerization of the denture basis. When SRM is fixed to already polymerized PMMA, the bond strength is higher $(0,94-2,56 \mathrm{MPa})$ [28]. This data is in contradiction to the results of Amin et al., who claim just the opposite in a similar research

Hatamlech et al. investigate the bond strength between Molloplast B and differently treated acryl surface (smooth, rough and fiber reinforced "Sticktech" surface). The highest values of bond strength were measured in the third group of 


\section{International Journal of Science and Research (IJSR) \\ ISSN (Online): 2319-7064}

Index Copernicus Value (2015): 78.96 | Impact Factor (2015): 6.391

samples. In this group cohesive fractures predominate. Between the results of the other two groups statistically significant difference is not found [29].

Many authors have investigated the influence of pulse duration and various energy levels of Er:YAG laser to the bond strength between PMMA surface and silicone SRM. The purpose of this interface micro roughening is achieving a better retention. Akin et al. investigate the effect of different way of treatment of the contact surface between silicone SRM and PMMA and its reflection to the bond strength. For that purpose, the authors use sandblasting apparatus, Er:YAG, Nd:YAG and KTP lasers. Tensile bond strength tests are done at a speed of $5 \mathrm{~mm} / \mathrm{min}$. The results show that treating of contact surface with Er:YAG laser leads to bond strength increase, while sandblasting reduces it [30,31]. Chung et al. and Usumez et al. in their own investigations also use sandblasting apparatus and claim that bond strength increases by this method [32,33]. A possible explanation could be the microgrooves that have been created and probably they are insufficient for the liner to penetrate into them. Another possible explanation is the stress concentration in the contact zone. The positive effect of Er:YAG laser is due to its greater energy. It is sufficient to create deeper and wider grooves, where the molecules of the relining material can be "locked in".

Jacobsen et al. used $\mathrm{CO}_{2}$ laser and claimed that is does not increase the strength between the materials of the test samples. According to the same research, treating of the acrylic contact surface with Er:YAG /10 Hz, 3 W, 300 mJ/ with long pulse duration (frequency) increases the tensile bond strength! The energy from the high energy impulse is enough to instantly evaporate the water molecules, which is accompanied with substantial increase of volume and in this way the contact surface increases. This enables the penetration of the liner in the microgrooves, increasing the bond strength [34]. The results of this research match the conclusion made by Delfino et al. [35]. Lawrence and Li determine that $\mathrm{Nd}$ :YAG laser expands the microgrooves on the PMMA contact surface from 1.95 to 2.22 micrometers and reduces the contact angle between the acrylic resin and the fluids [36].

\section{The influence of the primers and adhesives to the bond strength}

Primers play a crucial role in the bond strength between the test samples made of acrylic resin on one side and selfcuring silicone liners on the other. Minami et al. take under consideration the influence of the mechanical fatigue and the thermal factors. Their conclusion is that Sofreliner Primer for example gives better results in comparison to Reline Primer regarding the bond strength after thermo cycling and cyclic mechanical load.

Demir et al. report that during the peeling bond strength tests, thermo cycling has no influence on the bond strength between Molloplast $\mathbf{B}$ and the acrylic denture basis. A possible reason might be the Primo adhesive - a 3methacryloxypropyltrimethoxy sealant. One side of its molecule is linked to PMMA and the other to the VPS relining material. The authors declare the prevalence of cohesive fractures, which explains the high values of bond strength. The link between the SRM and putty PMMA is an effective way of increasing the values of the peeling bond strength tests [37].

The substance maleic anhydride /MA/ reacts with the contact surface of the acrylic denture base and increases the bond strength with the SRM. Despite the undeniable advantages of the silicone SRM, they can not create a sufficient bond with the PMMA denture base. That's why, they are supplied with an adhesive. In most of the cases it contains an organic dissolving agent and a monomer, which dissolves the acrylic base and reacts simultaneously with both materials [38]. The organic solvent affects the depth of penetration of the monomer and the reactivity of the added liner as well $[39,40]$. MA is a reactive monomer, containing unsaturated double bond and acid anhydride groups. The presence of these groups causes series of chemical reactions. Treating of the acrylic surface with MA or with some of its esters prior the adhesive application increases the bond strength. This improvement is due to the presence of anhydride groups providing the bond with the acrylic resin [41].

Maleic functional groups have a wide range of application. Similar chemical groups improve substantially bond strength /up to $4.84 \mathrm{MPa} /$. With $5 \%$ concentration of copolymer of maleic anhydride styrene, applied on similar test samples of polyamide and polystyrene, bond strength of $2.53 \mathrm{MPa}$ is achieved [42].

After treating of the contact surface of similar test samples made of PMMA and self-curing silicone liner with:

a) acetone for $30 \mathrm{sec}$.,

b) MMA monomer for $180 \mathrm{sec}$.,

c) methylene chloride for $15 \mathrm{sec}$ and submitted to 500 cycles $/ 5-55^{\circ} \mathrm{C} /$ the following results were obtained:

- MMA increases the bond strength and at the same time has little impact on the flexural strength of the denture base.

- Decreasing of the flexural strength is higher in the test samples treated with acetone!

The choice of a chemical agent, in order to increase the bond strength, should be carefully done, otherwise it may have a reverse effect [43]. Kaur, H. et K. Datta; Takahashi, Y. et J. Chai; Leles, C. et al.; Wieckiewicz, W. et al. also confirm that, the use of MMA monomer, dichloromethane, chloroform or acetone for preliminary treatment of the contact surface increases the bond strength and the treatment of the acrylic denture base with monomer decreases the microleakage.

\section{The effect of thermo cycling on the bond strength}

Thermo cycling is a commonly used method, which main purpose is to get simulated aging of the test samples. Along with the constant dipping into solutions with different temperature, usually between $5-55^{\circ} \mathrm{C}$ /a method used in rubber industry/, other methods for simulated aging are - the consecutive exposure to hot and cold air spray; long-lasting stay in distilled water; ultraviolet light radiation etc (fig.3).

\section{Volume 6 Issue 8, August 2017}




\section{International Journal of Science and Research (IJSR) ISSN (Online): 2319-7064}

Index Copernicus Value (2015): 78.96 | Impact Factor (2015): 6.391

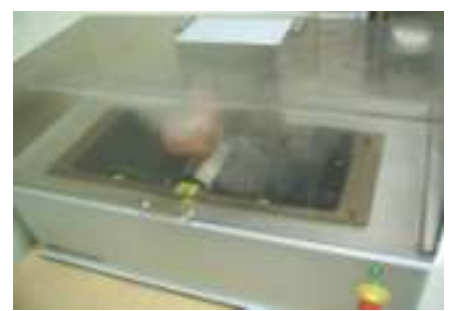

Figure 3: A thermo cycling device

Silicone based SRM are not seriously affected by the process of thermo cycling. Only cold-curing relining materials soften slightly, whilst the heat-curing materials keep their softness the same [44]. After thermo cycling the bond strength increases in some silicone SRM /Ufi Gel C/ and decreases in others /Molloplast B, Mollosil, Ufigel P/ [45]. Acrylic based SRM are not so stable and easily change their color, softness, absorption and cytotoxicity [46].

Madan, N. and K. Datta compare the changes in heat-curing and self-curing silicone liners from one and the same manufacturer $^{1}$ before and after thermo cycling. After 2500 cycles the bond strength increases in Mollosil /self-curing SRM/ and decreases in Molloplast B /heat-curing SRM/. Nevertheless the bond remains stronger in Molloplast B in comparison to Mollosil. This shows that the heat-curing relining materials have higher tensile strength module [47]. These statements are in agreement with the results of similar studies conducted by Kulak-Ozkan Y. et al.; Pinto J. et al [48, 49]. Craig et Gibbons; Dootz et al.; Emmer T. et al. state just the opposite - the long-lasting stay in water of all kinds of liners, regardless of their chemical composition and polymerization type, increases their bond strength with the denture base. They consider that a stable bond between the acrylic denture base and silicone liner is hard to achieve. Different behavior of random silicone SRM might be due to their various chemical composition. The polymerization type also affects the mechanical properties. On the other hand Elias and Enriques report of growing percentage of mixed /cohesive and adhesive/ fractures after thermo cycling.

In acrylic based relining materials after thermo cycling, bond strength increases, whilst in silicone based materials, thermo cycling has little effect on bond strength. According to Al-Athel et al. after thermo cycling the bond strength in silicone liners decreases /from 1.72 to $1.46 \mathrm{~N} / \mathrm{mm}^{2} /$. This statement is in contradiction to the results of Kulak-Ozkan et al. They even claim that after thermo cycling the bond strength increases /from 6.6 to $8.9 \mathrm{~kg} / \mathrm{cm}^{2} /$. Pinto at al. on the other hand report of no statistical difference in the obtained data from their experiments. Various results from different authors are due to the different methods they have used [50]! Simulated aging of the silicone samples has little effect on them, but increases the tensile bond strength. Acrylic relining materials have better initial softness, which unfortunately decreases in time [51].

${ }^{1}$ Detax GmbH \& Co. - Ettlingen, Germany

\section{Conclusion}

Factors like: immersion fluids, chemical composition, types of interface, various kinds of primers and adhesives, as well as aging, play a crucial role to the bond strength between denture basis and soft relining materials. The diversity of methods used by different authors is still a problem, because of the variety of results achieved and their correct interpretation. Obviously there is a great need of unification of the research methods.

\section{Acknowledgements}

This investigation was partially supported by University grant - DP-06/2013 of Medical University - Plovdiv, Bulgaria

\section{References}

[1] Moodhy, A. et R. Jagger. Effect of test method on the bond strength of a silicone resilient denture lining materia. J Prosthet Dent 1996; 76: 535-540.

[2] Aleksandrov, Sv. Study of the bond strength between a laboratory resin and a base dental alloy using micro and macro mechanical cutting tests. Doctoral dissertation. Medical University Plovdiv, 2015.

[3] Atanasov, Iv. Dental materials, Sofia Smile Centre, Sofia, 2013:39:43

[4] Dikova, C. Dental materials. Part II, Medical University Varna. 2015: 55

[5] Yankova, M., Yordanov, B. Application of elastic dental materials on a patient with partially and fully edentulous jaws. Infodent. 2014; 2:3-11.

[6] Aydin, A., H. Terzioglu, A. Akinay et al. Bond strength and failure analysis of lining materials to denture resin. Dental Materials. 1999; 15: 211.

[7] Kendall, K. The Adhesion and Surface Energy of Elastic Solids. J Phys D 1971; Appl Phys 4: 1186-1195.

[8] Lindley, P. Ozone Attack at a Rubber Metal Bond, J Instn Rubber Ind. 1971; 5: 243-248.

[9] Wright, P., Characterization of the Adhesion of Soft Lining Materials to Poly (methyl methacrylate). J. Dent. Restor. 1982; 61(8):1002-1005.

[10]Al Rifaiy, M. Shear bond strength between light polymerized hard reline resin and denture base resin subjected to long term water immersion. Saudi Dent J. 2012; 24: 23-7.

[11] Rajaganesh, N., S. Sabarinathan S, N. Azhagarasan et al. Comparative evaluation of shear bond strength of two different chairside soft liners to heat processed acrylic denture base resin: An in vitro study. J Pharm Bioall Sci. 2016; 8, Suppl S1:154-159.

[12] Naik, A. et J. Jabade. Comparison of tensile bond strength of resilient soft liners to denture base resins. J Indian Prosthodont Soc. 2005; 5: 86-88.

[13] Mutluay, M. et I. Ruyter. Evaluation of bond strength of soft relining materials to denture base polymers. Dent Mater. 2007; 23: 1373-1381.

[14] Braden, M., P. Wright, S. Parker. Soft lining materials: A review, Eur J Prosthodont Rest Dent. 1995; 3: 163 174.

[15] Sinobad, D., W. Murphy, R. Huggett et al. Bond strength and rupture properties of some soft denture liners. Journal of Oral Rehabililalion.1992; 19: 151-160.

[16] Webber, R., G. Ryge. The Determination of Tear Energy of Extensible Materials of Dental Interest. J Biomed Mater Res. 1968; 2: 281-296. 


\section{International Journal of Science and Research (IJSR) \\ ISSN (Online): 2319-7064}

Index Copernicus Value (2015): 78.96 | Impact Factor (2015): 6.391

[17] Yanikoglu, D., S. Denizoglu. The Effect of Different Solutions on the Bond Strength of Soft Lining Materials to Acrylic Resin. Dental Materials Journal. 2006; 25 (1): 39-44.

[18] Mese, A., K. Guzel, E. Uysal. Effect of Storage duration on tensile bond strength of acrylic or silicone-based soft denture liners to a processed denture base polymer. Acta Odontologica Scandinavica. 2005; 63: 31-35.

[19]Pisani M., C. Silva-Lovato, A. Malheiros-Segundo. Bond Strength and Degree of Infiltration between Acrylic Resin Denture Liner after Immersion in Effervescent Denture Cleanser. Journal of Prosthodontics. 2009; 18: 123-129.

[20] Landayan, J., A. Manaloto, J. Lee et al. Effect of aging on tear strength and cytotoxicity of soft denture lining materials; in vitro. J Adv Prosthodont. 2014; 6: 115120.

[21] Sarac Y., T. Basoglu, G. Ceylan et al. Effect of denture base surface pretreatment on microleakage of a silicone based resilient liner. J Prosthet Dent. 2004; 92 (3): $283-$ 287.

[22] Fowler, A., A comparison of bonding strength characteristics between a silicone rubber, Silastic 390, and various denture base resins. Master's thesis, Texas: University of Texas, 1968.

[23] Wood, W., D. Johnnson, M. Duncanson, Variables affecting silicone-poly(methyl methacrylate) interfacial bond strengths, J Prosthod. 1993; 2:13-18.

[24] Lassila, L., M. Mutluay, A. Tezvergil-Mutluay et al. Bond Strength of Soft Liners to Fiber-Reinforced Denture-Base Resin. Journal of Prosthodontics. 2010; 19: 620-624.

[25] Jagger, D., R. Jagger, S. Allen et al. An investigation into the transverse and impact strength of 'high strength' denture base acrylic resins. Journal of Oral Rehabilitation. 2002; 29: 263-267.

[26] Minami H. ,S. Suzuki, H. Ohashi et al. Effect of Surface Treatment on the Bonding of an Autopolymerizing Soft Denture Liner to a Denture Base Resin. Int $\mathbf{J}$ Prosthodont. 2004; 17: 297-301.

[27] Amin W., A. Fletcher, G.Ritchi. The nature of the interface between polymethyl methacrylate denture base materials and soft denture liners. J Dent 1981: 336-346.

[28] Kawano, F., E. Dootz, A. Koran III et al. Bond Strength of Six Soft Denture Liners Processed Against Polymerized and Unpolymerized Poly(methyl Methacryiate). Int J Prosthodont. 1997; 10: 178-182.

[29] Hatamleh, M., C. Maryan, N. Silikas et al. Effect of Net Fiber Reinforcement Surface Treatment on Soft Denture Liner Retention and Longevity. Journal of Prosthodontics. 2010; 19: 258-262.

[30] Akin, H., F. Tugut, B. Mutaf et al. Effect of different surface treatments on tensile bond strength of siliconebased soft denture liner. Lasers Med Sci. 2011; 26: 783788.

[31] Akin, H., F. Tugut, B. Mutaf et al. Strength of the bond between a silicone lining material and denture resin after Er:YAG laser treatments with different pulse durations and levels of energy. Lasers Med Sci. 2012; 27: 281-285.

[32] Chung, K., C. Chung, C. Chung et al. Effect of preprocessing surface treatments of acrylic teeth on bonding to the denture base. J Oral Rehabil. 2008, 35: $268-275$.

[33] Usumez, A, O. Inan, F. Aykent. Bond strength of a silicone lining material to alumina-abraded and lased denture resin. J Biomed Mater Res. Part B. 2004; Appl Biomater 71B: 196-200.

[34] Jacobsen, N., D. Mitchell, D. Johnson et al. Lased and sandblasted denture base surface preparations affecting resilient liner bonding. J Prosthet Dent 1997; 78: 153158.

[35]Delfino, C., W. Souza-Zaroni, S. Corona, et al. Microtensile bond strength of composite resin to human enamel prepared using erbium:yttrium aluminum garnet laser. J Biomed Mater Res. 2007; 80(2): 475-479.

[36] Lawrence, J., L. Li. Modification of the wettability characteristics of polymethyl methacrylate (PMMA) by means of $\mathrm{CO} 2, \mathrm{Nd}$ :YAG, excimer and high power diode laser radiation. Mater Sci Eng. 2001;303:142-149.

[37]Demir, H., A. Dogan, O. Dogan et al. Peel Bond Strength of Two Silicone Soft Liners to a Heat-cured Denture Base Resin. J Adhes Dent 2011; 13: 579-584.

[38] Dogan, O., G. Bolayir, A. Boztug et al. The effects of maleic anhydride terpolymer and its ester derivatives on tensile bond strength between the acrylic resin and resilient lining material. J Appl Polym Sci 2007; 104: 1338-1341.

[39] Arima, T., H. Nikawa, T. Hamada et al. Composition and effect of denture base resin surface primers for relineacrylic resins. Journal of Prosthetic Dentistry. 1996; 75: 457.

[40] Roth, J., V. Albrecht, M. Nitschke et al. Surface functionalization of silicone rubber for permanent adhesion improvement. Langmuir. 2008; 24: 1260312611.

[41] Turgut, M., G. Bolayir, O. Dogan et al. Bond strength of a silicone soft lining material to poly (methyl methacrylate) resin treated with maleic anhydride and its prepolymers. J Adhesion. 2007; 83: 927-938.

[42] Lee, Y., K. Char. Enhancements of interfacial adhesion between amorphous polyamide and polystrene by insitu copolymer formation at the interface. Macromolecules. 1994; 27: 2603-2606.

[43] Gupta, S. Effect of Surface Treatment on the Flexural Strength of Denture Base Resin and Tensile Strength of Auto polymerizing Silicone Based Denture Liner Bonded to Denture Base Resin. J Indian Prosthodont Soc. 2010; 10(4): 208-212.

[44] Hekimoglu, C., N. Anil. The effect of accelerated ageing on the mechanical properties of soft denture lining materials. Journal of Oral Rehabilitation. 1999; 26: 745-748.

[45] Sertgoz, A., Y. Kulak, H. Gedik et al. The effect of thermocycling on peel strength of six soft lining materials. Journal of Oral Rehabilitation. 2002; 29: 583-587.

[46] Park, S., Y. Lee, B. Lim et al. Changes in properties of short-term-use soft liners after thermocycling. Journal of Oral Rehabilitation. 2004; 31: 717-724.

[47] Madan, N., K. Datta. Evaluation of tensile bond strength of heat cure and auto polymerizing silicone-based resilient denture liners before and after thermo cycling. Indian Journal of Dental Research. 2012; 23(1): 64-68. 


\section{International Journal of Science and Research (IJSR) \\ ISSN (Online): 2319-7064}

Index Copernicus Value (2015): 78.96 | Impact Factor (2015): 6.391

[48] Kulak-Ozkan, Y., A. Sertgoz, H. Gedik. Effect of thermo cycling on tensile bond strength of 6 silicone based resilient denture liners. J Prosthet Dent 2003; 89: 303-310.

[49]Pinto, J., M. Mesquita, G. Henriques, M. Nobilo. Effect of thermo cycling on bond strength and elasticity of 4 long term soft denture liners. J Prosthet Dent. 2002; 88: 516-521.

[50] Kutay, O. Comparison of tensile and peel bond strengths of resilient liners. Journal of Prosthetic Dentistry. 1994; 71: 525.

[51] Takahashi, J., R. Consani, G. Henriques et al. Effect of Accelerated Aging on Permanent Deformation and Tensile Bond Strength of Auto polymerizing Soft Denture Liners. Journal of Prosthod. 2011; 20: 200204.

\section{Author Profile}

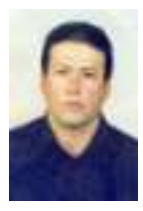

Dr Ilian Hristov, received his MSc Degree from Medical University - Plovdiv, Faculty of Dental Medicine in 1996. He worked for 4 years as a General dentist. From 2000 he is an assistant-professor and from 2016 is a PhD-student in the Department of Prosthodontics, Faculty of Dental Medicine, Medical University, Plovdiv, Bulgaria. He is an active member of the: Bulgarian Dental Union (BDU), Bulgarian Scientific Dental Association, Balkan Scientific Society (BaSS). He speaks English and Russian fluently. Faculty of Dental Medicine, Medical University, Plovdiv, Bulgaria. 3 Hristo Botev blvd., Plovdiv, Bulgaria 